\title{
Study on Acquisition of Lexical Chunks from the Perspective of the Zone of Proximal Development
}

\section{Chaowu Liu', Shuai Gao² and Dongmei Meng ${ }^{3}$}

\author{
${ }^{1}$ Foreign Languages School of East China Jiaotong University, Nanchang, Jiangxi 330000; \\ ${ }^{2}$ MTI Graduate School of Foreign Languages, East China Jiaotong University, Nanchang, Jiangxi \\ 330000; \\ ${ }^{3}$ Foreign Languages School of East China Jiaotong University, Nanchang, Jiangxi 330000.
}

\begin{abstract}
As an important part of linguistics, definition and development of lexical chunks, as well as progress of various application channels based on Practical Applied Linguistics and Pedagogy, is now more and more valued. Compared with traditional teaching methods, the lexical approach is more flexible and more scalable. In current teaching environment, taking lexical approach as a research field of zone of proximal development and putting them together can make lexical approach more promising in the future.
\end{abstract}

Keywords:Lexical chunks; Acquisition of lexical chunks; Zone of proximal development

\section{最近发展区视角的词块习得研究}

\author{
刘朝武 ${ }^{1}$, 高帅 ${ }^{2}$, 孟冬梅 ${ }^{3}$
}

(1. 华东交通大学外语学院 江西南昌 330000;

2.华东交通大学外语学院 MTI 研究生 江西南昌 330000;

3.华东交通大学外语学院 江西南昌 330000 )

摘要: 作为语言学重要的组成部分, 词块的定义, 发展, 以及多种应用渠道在现实应用语言学和教学法的基础上快速发 展, 目前受到了越来越大的重视。相比较传统教学法而言, 词块教学更具有一般教学法所不具备的灵活性, 延展性。在当下 的的教学环境下, 使词块教学纳入最近发展区的研究领域, 经过二者有机结合, 可以使词块教学在未来更具发展前景。

关键词: 词块; 词块习得; 最近发展区

中图分类号: H313 文献标志码: A

引言

经过研究分析母语使用者的记忆中所保留的语言记忆并非是由单个的单词组成, 而是介于句子和单词 之间的连贯的语言短语。在使用时根据语境将记忆中保留的语言短语经过适当增减以达到交流目的。 Michae1 Lewis 指出 “语言包含词块, 当词块组合在一起时就产生了连贯的语篇。语言交际主要靠典型的 词语搭配来表达意义, 运用搭配可以使人思维更快, 使交际效率更高。” ${ }^{[1]}$ 作为双语学习中的重要一部分, 词块的学习应用不仅可以改变二语学习者固有的语言学习方式, 改变其狂背单词却不擅应用的窘境, 还可 作为一种直观印象为学习者了解并加深印象。本文将简单介绍词块作为一种语言单位的具体内容, 进一步 探究词块教学的固有特点, 并结合 “最近发展区” 理论, 为词块教学作为一种教学方法开展的可能性进行 探讨。

\section{1 词块理论}

\section{1 词块定义}

词汇作为语言学习的基础, 其重要性不言而喻, 而在语言学习初期如果单纯依靠词汇记忆其结果往往 
不尽如人意。传统上来说语言习得过程呈阶梯状展开, 按照单词, 语句、语法、听力、口语依次进行。而 在上世界 70 年代中期, 以快速发展的语料库系统和语言学为基础, 大量关于语言习得的研究结果表明在 本民族语言使用者的记忆中存在大量成型或者半成型的语言模块, 使得本民族语言使用者在日常交流过程 中可以快速提取脑中的记忆板块。基于此类研究结果的出现, Becker（1975）年提出 “预制化语块” 的这 一概念, 他认为作为人际交往语言的最小单位， “预制化语块” 是人际交流之间信息的重要载体，而非单 个单词 ${ }^{[2]}$ 。而 Nattinger 和 DeCarrico (1992) 则认为这些语言模块是在语境中经过大量实践而逐步形成, 具有固定的形式，及相匹配的使用方式 ${ }^{[3]}$ 。Lewis（1992）提出 “Lexical Chunk” (词汇组块), 其出版的 The Lexical Approach 和 A Lexical Approach 为词块教学法奠定了理论基础。 ${ }^{[1]}$ 在其定义上 Wray 最早将 词块定义为 “一串预制的连贯或不连贯的词或其它意义单位，它整体存储在记忆中，使用时直接提取，无 需语法生成和分析 ${ }^{[4]}$ ”。而 Nattinger 和 DeCarrico 关于词块给出的解释是 “multi-word lexical phenomena that exist somewhat between the traditional poles of lexicon and syntax, conventionalized form / function composites that occur more frequently and have more idiomatically determined meaning than language that is put together each time $^{[3]}$. ”而具体分析 “词块” 可以看出, 词汇作为词块的基本组成形式，而块作为一个整体概念包含了词块中所包含的词组、 句型以及约定俗成的浐语、短句。

\section{2 词块的功用}

自从词块概念在语言学中被确立以来，其重要性已经被包括 leweis、Skehan、卫乃兴、罗明燕等中外 学者认可并进行大量研究。Pawley 和 Syder (1983) 更是在其专著中指出, 相较于英语母语使用者在日常交 际中的流利性, 对英语学习者的流利性程度提出了极高要求, 因此英语学习者必须熟练掌握运用大量词块 以实现这一目标 ${ }^{[5]}$ 。而 Altenberg 在其著作中表示, 作为承载语义的主题, 正常语际交流中的 $70 \%$ 是由预 制化模块即词块的完成 ${ }^{[6]}$, 而这一比重在 Nattinger 和 DeCarrico 的著作里是 $90 \%$ 。

二语学习者在学习过程中，始终面临 “质” 和 “量” 的问题：（1）如何像母语使用者一样逐步减少 淘汰那些语法完整措词正确却缺少地道意味的语句。（2）母语使用者的流利（Native-like fluency）记 忆中成千上万的词块, 需要时唾手可得 ${ }^{[7]}$ 。关于母语使用者记忆中数以千计的词块的特点可以被总结为以 下几点: (1) 结合语境, 母语使用者可以根据词块表达符合语境的流利表达。而正因为词块是根据语境中长 时间的实践形成, 因此母语使用者可以耗费极少的精力提取从而腾出更多的精力处理由词块承载的相关其 他相关信息中。（2）结合文化背景, 正是因为词块是在文化的影响下经过长时间的积淀而形成, 因此母语 使用者可以用词块作出更符合文化传统的语句, 例如:Frank is a man eating no fish, so we can believe in and depend on him. 句子的愿意是弗兰克是个诚实的人，我们可以信任并依赖他。原句中，eating no fish 作为理解句意的关键词其意义并非不吃鱼, 而是引申出英国传统文化中的宗教含义, 意为诚实的。而 如果把本句改下一下: Frank is an honest man, so we can believe in and depend on him. 其意虽然 没变, 其味却寡淡的多, 远不如 Eating no fish 来的更为传神和地道。而作为口语交际的现实考量, Widdowson 给出的概念是: “在现有的大量模块化型式、固定框架和语法规则下, 作出适量调整以迎合语 境的需求 ${ }^{[8]}$ 。”而 Norbert Schmitt 在其著作 Formulaic Sequences 中指出: 词块的受众群体的如此之 大是与交际功能用语密切相关 ${ }^{[9]}$ 。在口语实例中, “it is a long story” 用来作为故事或者对话的开端, 
而 “make yourself at home” 则用语主客之间的客气对话。从这些实例中不难看出, 词块作为实现语 用功能的重要一环, 其所承载的意义以极为简短可靠的方式保存下来。由于与功能用语的密切联系, 使得 词块称为二语学习者提高语言应用能力 (Pragmatic Competence) 关键的一环。总前所述, 大量词块的熟 练运用可以有效的提高二语学习者熟练性, 使其在语境交流环境中迅速做出合理表达和运用。

\section{3 词块的分类}

George A. Miller 于 1956 年发表了 《7 2 , 我们加工信息能力的局限》这一论文, 在文中, 他着重 探讨了人一般短时记忆为 7, 并有可能在 5-9 之间浮动, 但这一论断并不适用在由短时记忆大量重复而形 成的长时记忆上。由此可见, 如果将日常所用词块按照词性、用途、与已联系、内部功能分组的话, 将极 大地提高词块的可记忆性（Memorability）, 依次降低复杂词块的学习难度, 使得降低语言习得的难度和 语言使用的门槛。

根据词块在语际交流中的定位，Zenick 和 Dyer（1987）规范了词块应具有相当规模的整体构架：（1） 词汇;（2）固定的模块;（3）在交流中可能有所变化的词块;（4）拥有整体架构并包含固定形式的词块。 ${ }^{[10]}$ 而在所有学者的分类中, Nattinger, DeCaricco 和 lewis 的分类更广为人知, Nattinger 和 DeCaricco （1992）从衔接的角度将常用词块分为四类：(1) 多元词块, 由超过一个单词所组成的固定短语搭配, 如 be good at; (2) 固定表达词块, 经过长期时间而在语境中形成的固定用法, 如 how is it going ? 和 it is reported that……（3) 限制性词块, 意指通常在句式中随着语境而变化的词块, 如 be good for; (4) 句 型构造词块, 在句子中上启转折的词块及句子架构, 如 either $\cdots \mathrm{or}^{[3]}$ 。而根据词块的基本形式, 李维斯 (Lewis) 将常用词汇组合分为四项基本类型: 1 词汇短语, 如 al though 或是 on the other hand 等。本类 型作为英语学习者所需掌握的必要形式, 包含了应该熟记的基本词汇和相关内容; 2 高频率搭配词, 如 on the basis of 或 out of breath 等。在特定的语言中，熟练运用语言者熟知词汇之间的搭配，懂得把把词 汇串联在一起, 形成固有意义; 3 约定俗成的惯用语, 如 It is we11-known to us that 或 It is a common belief that 此类是指在某一语境文化中, 语言在经过长时间的实践而形成的固定表达形式, 此类型通常 是具有一定语法意义的整体语句; 4 句式框架, 例如 first of all that …则属于简单的语言结构性叙事 方式, 是一类简单的语言叙述形式 ${ }^{[11]}$ 。

\section{2 词块教学的作用}

根据显示的效果而言, 严格建立在语法教学上的传统教学, 在面临日益严峻的竞争压力下显得力有不 逮。究其原因, 无非是传统之下的教学过于强调语法而对词汇重视不足, 而这种重语法轻词汇的教学模式 也极易让二语初级习者摸不着头脑。事实上, 这种方向性和倾斜如果在探究词汇和语法深层次的联系之后 完全可以避免。Michael Lewis 认为 “词汇与语法之间并无必要的差异, 恰恰相反, 二者之间存在密不可 分的联系” [1]。在 Holliday 的词汇-语法论里还提出, “词汇作为最精密的语法” 这一论点, 这一论点的 核心在于系统的阐述了词汇和语法的的相互关系: 二者并不质的区别, 显存的差异仅是量的不同的表象 ${ }^{[12]}$ 。 而根据对语料库的分析, 自然对话中, 绝大部分的语义是由词块完成, 而作为双向性的概念, 如果将词块 导入教学活动中, 则可让二语习者的学习活动更具灵活性。

(1) 有助于培养语感

二语学者, 以英语四级为标准, 还停留在一个较为基础的阶段, 在这个阶段如果过分强调语法的投入, 
则会造成主干强而躯体弱的若态。而更有专家学者Widdowson（1989）就论证过语法和词汇的关系：“词 汇比语法更加重要, 语言知识在某种程度上可以看作词块的只是, 而语法则屈居次位, 在语言中充当调整 和协助的角色” ${ }^{[9]}$ 。在这种情况下, 适当引入词块教学, 则可引导二语学习者协调发展, 有助于培养学习 语的语感。众所周知, 语感的是二语学习者对语言认识的投射, 语感的形成及健全程度对学习者语言流利 输出是否地道有着至关重要的关系。因为词块是半成型的语言模块, 其本身固有语言结构、语法限制和适 用方式, 在二语初级学者的学习过程中, 可以为之提供较为直观语感养成方式。

(2) 有助于克服母语的重度依赖

在二语学习过程中, 学习者将不可避免的受到母语和学习与逻辑碰撞的影响, 这种情况被称作语言的 负迁移，而语言的负迁移现象是普遍存在的。而汉语重意合，英语更偏重型合，二者之间很难找到逻辑上 基本对等的词汇, 所以众多二语初级学者将不可避免的在母语的影响下进行错误的语言输出:Because it was raining, so we stayed at home. 这个句子在汉语逻辑里面是没有问题，“因为天下雨了，所以我们 待在家里。而在英语中, because 和 so, 不能连用, 正因为英汉双语中的逻辑词汇不对等, 而二语习者的 出发角度仍是母语思维，才造成这种问题的出现。如果在二语习者提前摄入大量词块的情况下，无论在口 语或者书面语言中，上述问题都能得到明显的改善。日常口语的特点无外乎 “短平快” ，是在有限的词汇 表达中传递出大量信息。而从词块的特点 Analyticity（可分析化）和 Formulaicity（公式化）出发，词 块中含有大量预置模块, 这些模块作为经过大量实践的客观存在, 不需要考虑句法结构具有易上手, 易适 用的特点, 如 I got it, easy does it 等。可以迅速满足适用者的需要。而针对于书面语言中, 胡小颖 (2009) 在观察中发现, 学生掌握的词块越多, 作文的题目越高 ${ }^{[13]}$ 。针对于二语学者的负迁移, 可以加强 适用英语中的, it is said 表起, furthermore 表更深层次的阐述, on the contrary 表句意的转折, above al1 表结束。从长久来看, 英语中的名人浐语, 书中的警局格言都可以作为词块的补充部分进行收录, 从 而进一步扩展脑中的词块库, 如果二语学者能够熟练运用以上的词块进行书面写做, 相信可以使文章清晰 更有条理，通过被词块改善过的外语思维表达的语句也将更加地道。

（3）词块的可扩充性和规范性

作为二语习者的重要目标, 英语四级对词汇的要求是保持在 4500 左右, 与此同时, 在词汇难度上也 有所提升, 这使得拥有一定基础的二语习者处于语言学习的关键性阶段。而人的记忆曲线在呈 M 状提升时, 应尤为注意在学习过程中可能出现的负面影响, 如母语的负迁移和高原现象, 长此以往, 容易加重学习者 的焦虑情绪但如果能够有针对性的加强词汇练习, 提高词汇记忆, 这种情况亦将得到改善。而根据《英语 作文中的词块使用特征研究》 ${ }^{[14]}$ 及 《70 小时攻克攻克核心英语词块》 ${ }^{[15]}$ 及语料库的统计分析, 以英语专业 四级所包含的词块数目为标准, 在此区间, 只存在不超过 1500 个核心词块, 二语习者通过吸收上述所包 含的大量词块, 不仅可以扩充词汇量, 还可以使语言输出更为规范、地道, 更能在潜移默化中自我约束, 培养语感。

\section{3 最近发展区视角的词块习得}

上世界三十年代，维果斯基（Vygotsky）提出 “最近发展区” 这一概念，在这一概念中，他系统的将 学习者学习过程和学习目标划分为两个相关领域。即: 一是现阶段已经可以确保的事实发展水平（actual development leve1) 是现有水平足以独立完成同一层次的问题。二是潜力发展水平（level of potential 
development）主要是指学习者在老师或相对层次较高的人的配合帮助下提高水平, 解决较高层次的相对 应问题。 ${ }^{[16]}$ 而金格 (Kinger) 则进一步阐述这一理论, 他认为在最近发展区中具有两个突出特征，一是学 习者的任务须在当前水平和潜力水平之间的区间, 如果超出太多, 则极易损伤学习者的积极性; 二是在学 习者过程之中，必须要有相对层次更高的人起到规划框架，启迪鼓励的作用 ${ }^{[17]}$ 。

\section{1 基于支架式教学的词块教学设计}

支架式教学法是以建构学习注意理论为基础提出的一种以学习者为中心，以培养学生的问题解决能力 和自主学习能力为目标的教学法。该教学法是指一步一步地为学生的学习提供适当的、小步调的线索或提 示 (支架), 让学生通过这些支架一步一步的攀升, 逐渐发现和解决学习中的问题, 掌握所要学习的知识, 提高问题解决能力。如果以上述理论为基础, 老师或水平层次较高者以训练、提升、巩固为指导思想, 激 起学习者求知欲望, 并指导学习方向, 将词块分类、排序, 嵌入到学习者的整体目标中, 有限度参与学习 者的学习过程, 既不过分突出己方影响, 又不致严重干扰学习者的学习热情。而在整个过程中, 建立一套 标准的评估体系将对审核学习者的提升水平, 评估教学效果和增强学习者信息, 加强学习动力至关重要。

\section{2 最近发展区的干预}

(1) 认知发展区的干预

认知螺旋上升方式是构设英语课程教学顺序的一种方式，是以英语课堂教学发展连续统为背景，对主 体 (师生) 在外语教学中进行的各种主题或技能 (主要包括动机、目标语、准言语交际) 的轮回提升的信息流 程的整体描述 (如前所述)。螺旋的周围是不同的主题和不同类型的技能 (词汇、语音、言语交际、听力、 阅读), 课程是穿越每一主题和技能的螺旋系列, 扩展表示的是在每一个水平上包纳先前的学习。螺旋中 的每一环所含目标的层次和复杂水平不断增加。随着教学的进展, 这些主题或技能将在后续的更高水平上 进一步巩固或提升。

大学英语词块教学的螺旋上升模式决定了不同学业阶段教学项目的重心必须合理安排, 而且不同层面应该 有不同的侧重点，具体如下：

表 1 认知发展区的螺旋上升干预

\begin{tabular}{|c|c|c|}
\hline 螺旋层面 & 项目 & 侧重点 \\
\hline \multirow{5}{*}{$\begin{array}{l}\text { 螺旋底层 } \\
\text { (第一、二学期) }\end{array}$} & \multirow{3}{*}{$\begin{array}{l}\text { 认 } \\
\text { 知 }\end{array}$} & 语音; 单词和短语的认知与熟知; 构词; 快速阅读; 语法的普遍规则和特殊情况 \\
\hline & & $\begin{array}{l}\text { 基本语言运用 (会话和写作); 诱导学生对语篇或人物角色的基本分析; 以角色再现的方式巩固学 } \\
\text { 生对语篇或人物角色的把握并借此逐步训练学习者语言应用能力 }\end{array}$ \\
\hline & & 引导和培养学习者以语言应用为导向的二语习得理念和能力，逐步培养学习者良性的认知风格 \\
\hline & \multirow[b]{2}{*}{$\begin{array}{l}\text { 情 } \\
\text { 感 }\end{array}$} & 在意识上, 扭转学生学习理念, 坚立良性的语言学习目的和观念 \\
\hline & & $\begin{array}{l}\text { 在实践上, 通过增加教学活动的趣味性和多样性以及降低教学内容的难度来重点培养学习者自信 } \\
\text { 心和学习兴趣; 加强情感激励的介入 }\end{array}$ \\
\hline \multirow{3}{*}{$\begin{array}{l}\text { 螺旋中层 } \\
\text { (第二、三学期) }\end{array}$} & \multirow[b]{2}{*}{$\begin{array}{l}\text { 认 } \\
\text { 知 }\end{array}$} & $\begin{array}{c}\text { 语音的逐步巩固; 单词和短语的熟知与运用; 构词的模块学习; 阅读技巧 (精读与泛读的训练) ; } \\
\text { 语法的难点分析; 基本翻译技巧的指导与训练 }\end{array}$ \\
\hline & & $\begin{array}{l}\text { 语言的灵活运用; 以设问和讨论的方式增强学生对语篇或人物角色的分析能力; 以内容、情节或题 } \\
\text { 材改编等方式加强学生对语篇或人物角色的把 } \\
\text { 握并借此增强学生的语言灵活应用能力 }\end{array}$ \\
\hline & $\begin{array}{l}\text { 情 } \\
\text { 感 }\end{array}$ & $\begin{array}{l}\text { 继续提高学习兴趣; 一定程度上增加教学活动的竞争性来培养成就感以提高动机内化 } \\
\text { 的内摄调节和认同调节程度 }\end{array}$ \\
\hline \multirow{2}{*}{$\begin{array}{l}\text { 螺旋高层 } \\
\text { (第四学期) }\end{array}$} & 认知 & $\begin{array}{l}\text { 以辩论等方式进行听、说、读、写、译等综合能 } \\
\text { 力的培养与训练; 以 presentation 等方式进行自主学习与分析能力的培养与训练 }\end{array}$ \\
\hline & 情感 & 继续以学业的成效加强认同性动机与自我的其他方面的整合 \\
\hline
\end{tabular}

（2）目标与任务制定中的干预

目标和任务的制定是以认知螺旋上升模式为指导。本实证教学展示内容以《现代大学英语》第一册 (北 
京：外语教学与研究出版社, 2012) 为示范。

\begin{tabular}{|c|c|c|c|c|}
\hline 项目 & 内容 & 表现形式 & 原则 & 介入策略 \\
\hline $\begin{array}{l}\text { 长远 } \\
\text { 目标 }\end{array}$ & $\begin{array}{l}\text { 提高英语语言综合运用能 } \\
\text { 力, 尤其听说等应用能力。 }\end{array}$ & $\begin{array}{l}\text { 教师为主 } \\
\text { 学生参与 }\end{array}$ & $\begin{array}{l}\text { 符合社会和大 } \\
\text { 环境发展需要 }\end{array}$ & $\begin{array}{l}\text { 1. 榜样激励; 价 } \\
\text { 值与信心激励 } \\
\text { 2. 得到学生认可 } \\
\text { 3. 把学习价值与 } \\
\text { 动机强度相匹配 }\end{array}$ \\
\hline $\begin{array}{l}\text { 学期 } \\
\text { 目标 }\end{array}$ & $\begin{array}{l}\text { 本学期主要是盘活学生中学 } \\
\text { 所学内容, 培养学生良好的 } \\
\text { 学习习惯, 激发学生英语学 } \\
\text { 习的兴趣与信心, 努力使学 } \\
\text { 生明确自己的学习目的, 从 } \\
\text { 而更加积极有效地开展各种 } \\
\text { 学习活动。另外, 此学期特 } \\
\text { 别注重提高学生的语音, 巩 } \\
\text { 固语法知识, 扩大词汇量, } \\
\text { 提高阅读速度。 }\end{array}$ & $\begin{array}{l}\text { 教师为主 } \\
\text { 学生参与 }\end{array}$ & $\begin{array}{l}\text { 务实的、具体 } \\
\text { 的、循序渐进式 } \\
\text { 的 }\end{array}$ & $\begin{array}{l}\text { 1. 得到学生认可 } \\
\text { 2. 榜样激励 } \\
\text { 3. 信心激励 }\end{array}$ \\
\hline 近期 & $\begin{array}{l}\text { 注重提高学生的语音、语调 } \\
\text { 等基本语言能力, 提高句法、 } \\
\text { 语篇的基本分析能力, 通过 } \\
\text { Half a Day 的课文分析, 增 } \\
\text { 强记叙文的要素特征识别能 } \\
\text { 力。 }\end{array}$ & 师生共同参与 & $\begin{array}{l}\text { 现实的、具体 } \\
\text { 的、阶段性的、 } \\
\text { 台阶式的 }\end{array}$ & $\begin{array}{l}\text { 1. 因人而异, 难 } \\
\text { 度适中地采取 } \\
\text { 目标激励策略 } \\
\text { 增加成就感 } \\
\text { 2. 激励其不断追 } \\
\text { 求新的成就 } \\
\text { 3. 对学生有吸引 } \\
\text { 力 }\end{array}$ \\
\hline
\end{tabular}

课堂任务以学生的初始状态为依据, 以短期的易于达到的目标为主要特征, 这样不但能使学生了解自 己的实际水平, 在学习中不断检测自己的 “战绩”, 同时还可以在不断体味成就感的同时产生自主练习的 动机, 保障健康的学习导向; 在课堂内容的选择上主要以中等难度的内容为主, 允许在学习大纲允许的范 围内自由选择学习内容与进度, 并且通过不同层次的对比激发学生的积极性, 这样就会使学生对要学习内 容产生立体感和预见性, 清楚自己在 “学习线路” 所处的位置, 并能进行正确的自我分析与评价, 及时发 现学习内容的 “触发点”, 以保持高昂的学习热情和极大的学习兴趣; 在授课方式上, 以多样化的小组合 作创新为主, 降低了学生个体间的差异, 不但使他们有归属感和胜任感, 还会使他们学会从群体出发, 求 
同存异, 培养集体主义和团结协作的精神。同时, 良好的学习氛围会拓展学生的学习思路, 激发他们的学 习潜能，培养创造力和自我效能感。

二语学习者之间存在着基础水平, 学习背景和思维方式之间的差异如何找准学习者的最近发展区将是 老师所面临的一道显示难题, 鉴于此类问题的出现, 老师可以通过对学习者学业能力的基础测试, 对比研 究学习者的课业训练以完成对于学习者词块掌握程度乃至语言水平的初步统计。此外, 此外老师还可通过 对于学习者现有的知识储备以及将要进行的学习方向进行跟踪观察, 并辅以相近的有关词块进行针对性训 练, 通过开展学习结构, 进行速度来摸索出学生合理的最近发展区。以中学英语为例, 学习者在学到 turn 这个单词时, 在熟练掌握单词的基础上, 老师可以适时推出含有 turn 的词组如 “turn on 和 turnoff”, 此类词组贴近生活, 易于掌握。与此同时, 为了更好的有的放矢, 针对性、阶梯型教学即可保证各种程度 的学习者学有所获, 也可以促进整体进步, 促进最近发展区水平和实际学业水平相互转换, 并以此为基础 成就新的最近发展区这一良性循环。

\section{4 结论}

随着近二十年的迅速发展, 词块教学法受到了越来越多的瞩目, 作为对于教学理念的勇敢尝试, 词块 教学法无论在促进二语学习者的思维逻辑能力, 语言交流和文字输出的提高上都有着可圈可点的表现。而 所有理论都要在实践中得到进一步的发展和巩固。词块教学和最近发展区理论的有效结合, 将会在更大程 度上提高学习者的积极性, 培养他们的学习兴趣, 进而促成兴趣 $\rightarrow$ 学习 $\rightarrow$ 提高 $\rightarrow$ 兴趣这一良性循环, 而随 着学习者的培养过程, 二者结合这一教学方法还将得到进一步的巩固提高, 以便于更好的为二语学习者服 务。

\section{5 致谢}

本论文得到江西省社科规划项目 (15WX208) 的资金资助，感谢同仁，同事和家人的支持。

\section{Acknowledgements}

\section{This paper is funded by Jiangxi Provincial Social Science Program (15WX208).}

\section{参考文献:}

[1] Lewis M. The Lexical Approach [M]. Cambridge: Cambridge University Press, 1986.

[2] Becker, J. D. The Phrasal Lexicon [C]// Nash-Webber. B. and R. Schank (eds) . Cambridge Mass Theoretical Issues in Natural Language Processing. Bolt, Beranek, and Newman, 1975.

[3] Nattinger, J. and J. Decarrico. Lexical Phrases and Language Teachin[M]. Oxford: 0xford University Press, 1992.

[4] Wray, A. Formulaic Language and the Lexicon [M]. Cambridge: CUP, 2002.

[5] Pawley, A. and F. Syder. Two Puzzles for Linguistics Theory: Native-1ike Selection and Native-1ike Fluency [C]// Richards, J. and R. Schmidt. Language and Communication. London: Longman, 1983.

[6] Altenberg B. On the Phraseology of Spoken English: The Evidence of Recurrent Word-combinations [C]// Cowie, A. P. Phraseology: Theory, Analysis and Application. 0xford: 0xford University Press, 1998.

[7] 桂诗春. 我国外语教学的新思考 [J]. 外国语, 2004(4)：2-9.

[8] Widdowson, H. Knowledge of Language and Ability for Use[ J]. Applied Linguistics 10 ( 1989) : 128-137. 
[9] Schmitt, N. Formulaic Sequences: Acquisition Processing and Use [M]. Amsterdam: John Benjamins Publishing, 2004.

[10] Zernick, U. and M. Dyer. The Self-extending Phrasal Lexicon[J]. Computational Linguistics, 1987, (13): $308-327$.

[11] Lewis, M. Implementing the Lexical Approach: Putting Theory into Practice[M]. Hove: Language Teaching Publications, 1997.

[12] Holliday, Michael. Cohesion in English[M]. Beijing: Foreign Language Teaching and Research Press, 2001.

[13] 胡小颖. 词块法对二语写作能力发展的影响之实证研究 [J]. 疯狂英语（教师版），2009(02)：19-23.

[14] 70 小时攻克核心英语词块. 2015.6 世界知识出版社

[15]英语作文中的词块使用特征研究. 2016. 5 山东大学出版社

[16] Brown H. D. Teaching by Principles[M]. Upper Saddle River NJ. Prentice Hall Regents 1994

[17] Celeste Kinger Proximal Development in US Foreign Language Education [J]. Applied Linguistics 2002 (2) :240-261

联系人: 孟冬梅, 电话 13732978215, 邮箱 605840009@qq. com

\section{References:}

[1] Lewis M. The Lexical Approach [M]. Cambridge: Cambridge University Press, 1986.

[2] Becker, J. D. The Phrasal Lexicon [C]// Nash-Webber. B. and R. Schank (eds). Cambridge Mass Theoretical Issues in Natural Language Processing. Bolt, Beranek, and Newman, 1975.

[3] Nattinger, J. and J. Decarrico. Lexical Phrases and Language Teachin [M]. Oxford: Oxford University Press, 1992.

[4] Wray, A. Formulaic Language and the Lexicon [M]. Cambridge: CUP, 2002.

[5] Pawley, A. and F. Syder. Two Puzzles for Linguistics Theory: Native-like Selection and Native-like Fluency [C]// Richards, J. and R. Schmidt. Language and Communication. London: Longman, 1983.

[6] Altenberg B. On the Phraseology of Spoken English: The Evidence of Recurrent Word-combinations [C]// Cowie, A. P. Phraseology: Theory, Analysis and Application. Oxford: Oxford University Press, 1998.

[7] Gui Shichun. New Thoughts on Foreign Language Teaching in China [J]. Foreign Language, 2004(4): 2-9.

[8] Widdowson, H. Knowledge of Language and Ability for Use [J]. Applied Linguistics 10 (1989): $128-137$.

[9] Schmitt, N. Formulaic Sequences: Acquisition Processing and Use [M]. Amsterdam: John Benjamins Publishing, 2004.

[10] Zernick, U. and M. Dyer. The Self-extending Phrasal Lexicon [J]. Computational Linguistics, 1987, (13): 308-327.

[11] Lewis, M. Implementing the Lexical Approach: Putting Theory into Practice [M]. Hove: Language Teaching Publications, 1997.

[12] Holliday, Michael. Cohesion in English [M]. Beijing: Foreign Language Teaching and Research Press, 2001.

[13] Hu Xiaoying. An Empirical Study on Influence of Lexical Chunks Method on Development of Second Language Writing Ability [J]. Crazy English (Teacher Edition), 2009(02): 19-23.

[14] 70 Hours to Overcome Core English Lexical Chunks. World Affairs Press, 2015.6.

[15] A Study on Characteristics of Lexical Use in English Writing. Shandong University Press, 2016.5. 
[16] Brown H.D. Teaching by Principles [M]. Upper Saddle River NJ. Prentice Hall Regents 1994

[17] Celeste Kinger Proximal Development in US Foreign Language Education [J]. Applied Linguistics 2002 (2):240-261

Contact Person: Dongmei Meng, Tel: 13732978215, Email:605840009@qq.com 